\title{
Coupled Dynamics and Quiescent Phases
}

\author{
Karl P. Hadeler ${ }^{1}$ and Thomas Hillen ${ }^{2}$ \\ 1 Universität Tübingen, Germany \& Arizona State University \\ hadeler@uni-tuebingen.de \\ 2 University of Alberta thillen@ualberta.ca
}

Summary. We analyze diffusively coupled dynamical systems, which are constructed from two dynamical systems in continuous time by switching between the two dynamics. If one of the vector fields is zero we call it a quiescent phase. We present a detailed analysis of coupled systems and of systems with quiescent phase and we prove results on scaling limits, singular perturbations, attractors, gradient fields, stability of stationary points and amplitudes of periodic orbits. In particular we show that introducing a quiescent phase is always stabilizing.

\section{Introduction}

Two different dynamics acting on the same space can be coupled in several ways, e.g. by using the Lie-Trotter approach of periodically switching between the two dynamics or by diffusive coupling. Diffusive coupling has the advantage that the resulting system is autonomous. Whereas the limiting system of the Lie-Trotter approach leads to a convex combination of the two vector fields, diffusive coupling leads to another limiting system. In the general non-linear situation the two limiting systems are not equivalent. Here diffusive coupling is studied in terms of singular perturbation theory and in terms of second order systems.

If one of the two vector fields vanishes then a given dynamics is coupled to a quiescent phase. While one could conjecture that adding a quiescent phase should have similar effects as a delay, e.g. cause oscillations in negative feedback situations, the opposite is true. Introducing quiescent phases damps oscillations or even causes them to disappear. Quiescent phases occur in population models in various ways and under various names such as quiescent state [19], [12], dormancy [9], [17], resting phase [6], ecological refuge. It is generally understood that such phases may have drastic effects on the dynamics.

We show a general linear stability theorem for systems with quiescent phases near an equilibrium, another theorem on how a quiescent phase decreases the amplitude of a periodic orbit away from equilibrium, and we show 
some preliminary results on the global behavior of such systems. To our best knowledge these results are new.

In section 2 we compare diffusive coupling to the Lie-Trotter approach, establish the connection to second order equations, and give examples. We cast the problem into the framework of singular perturbations in section 2.3 and prove results on global behavior in section 2.4. In section 3 we introduce the concept of quiescent phases, study local stability of stationary points in section 3.1 , then periodic orbits in section 3.2, and global behavior of systems with quiescent phases in section 3.3. Finally, in section 4 we prove the generalized Lie-Trotter approximation (Theorem 1).

\section{Coupled Systems}

Let $f$ and $g$ be smooth vector fields on $\mathbb{R}^{m}$. The differential equations

$$
\dot{v}=f(v), \quad \dot{w}=g(w)
$$

have unique local solutions. One way to couple these two equations is the classical Lie product formula, the other is diffusive coupling. Here we compare both approaches.

\subsection{The Lie-Trotter Approach}

Let $\chi: \mathbb{R} \rightarrow[0,1]$ be a piecewise continuous function of period 1 . For $\delta>0$ consider the periodic system with period $\delta$

$$
\dot{u}_{\delta}=\chi(t / \delta) f\left(u_{\delta}\right)+(1-\chi(t / \delta)) g\left(u_{\delta}\right)
$$

with the initial datum $u_{\delta}(0)=u_{0}$. For a fixed time horizon $T$ consider the solution $u_{\delta}(t)$ for $0 \leq t \leq T$ for $\delta \rightarrow 0$. The limiting function $u(t)$ satisfies the autonomous equation

$$
\dot{u}=\rho_{1} f(u)+\rho_{2} g(u)
$$

with $\rho_{1}=\int_{0}^{1} \chi(s) d s, \rho_{2}=1-\rho_{1}$, and the initial datum $u(0)=u_{0}$. Although this statement it intuitively obvious, the proof is not that obvious, in particular as the important special case, i.e., the Lie product formula for matrices $\exp \{A+B\}=\lim _{k \rightarrow \infty}(\exp \{A / k\} \exp \{B / k\})^{k}$ (Lie 1875) is usually proved via estimates for matrix products, see [1], p. 254, and [8], p. 496. In the following \|\| is the maximum norm on $\mathbb{R}^{m}$ and the corresponding operator norm.

Theorem 1. Let $\|f(u)\|,\|g(u)\|,\left\|f^{\prime}(u)\right\|,\left\|g^{\prime}(u)\right\|$ be bounded by some constant $M$ uniformly in $u$. Let $T>0$ be a fixed time. Then for $\delta \rightarrow 0$ the solutions of (1) converge to the solution of (2) uniformly on $[0, T]$. 
The proof is given in Section 4. A special case arises for

$$
\chi(t)=\left\{\begin{array}{l}
1,0 \leq t<\rho_{1} \\
0, \rho_{1} \leq t<1
\end{array}\right.
$$

with some $\rho_{1} \in(0,1)$ and $\rho_{2}=1-\rho_{1}$. The vector field $(2)$ is a convex combination of the vector fields $f$ and $g$. The standard case is $\rho_{1}=1 / 2$. Extensions to infinite dimensions and operator semigroups are called LieTrotter and Trotter-Kato formulae (Trotter 1959, Kato 1978). For extensions to infinite-dimensional non-linear systems see, e.g. [15]. The Lie-Trotter approach is well suited for numerical schemes (e.g. fractional steps, alternating directions (ADI), splitting methods) because it simplifies the design of consistent schemes. From the view point of dynamical systems it has the disadvantage that the coupled system becomes non-autonomous.

The theorem can be generalized to the case of $N \geq 2$ vector fields $f_{i}$ in $R^{m}, i=1, \ldots, N$. Given piece-wise continuous functions $\chi_{i}$ which are periodic with period 1 , and $\chi_{i} \geq 0, \sum_{k=1} \chi_{k}=1$, Lie-Trotter coupling

$$
\dot{u}_{\delta}=\sum_{i=1}^{N} \chi_{i}(t / \delta) f_{i}\left(u_{\delta}\right)
$$

leads to the limiting equation

$$
\dot{u}=\sum_{i=1}^{N} \rho_{i} f_{i}(u) \quad \text { with } \quad \rho_{i}=\int_{0}^{1} \chi_{i}(s) d s .
$$

\subsection{Diffusive Coupling}

Let $\gamma_{1}, \gamma_{2}$ be positive coupling constants. Then consider the system in $\mathbb{R}^{2 m}$

$$
\begin{gathered}
\dot{v}=f(v)-\gamma_{2} v+\gamma_{1} w \\
\dot{w}=g(w)-\gamma_{1} w+\gamma_{2} v .
\end{gathered}
$$

With particle density $u$ and flux $z$ as new variables

$$
u=v+w, \quad z=\gamma_{2} v-\gamma_{1} w
$$

the system assumes the form

$$
\begin{aligned}
\dot{u} & =f\left(\rho_{1} u+\tau z\right)+g\left(\rho_{2} u-\tau z\right) \\
\tau \dot{z} & =\rho_{2} f\left(\rho_{1} u+\tau z\right)-\rho_{1} g\left(\rho_{2} u-\tau z\right)-z
\end{aligned}
$$

where the time constant $\tau$ and proportions $\rho_{1}, \rho_{2}$, with $\rho_{1}+\rho_{2}=1$, are given by

$$
\tau=1 /\left(\gamma_{1}+\gamma_{2}\right), \quad \rho_{i}=\tau \gamma_{i}, \quad i=1,2
$$


If we let $\tau$ go to zero then we arrive at the limiting system

$$
\dot{u}=f\left(\rho_{1} u\right)+g\left(\rho_{2} u\right)
$$

which is rather different from the limiting equation (2). The equations (6) and (2) are equivalent if at least one of the functions $f, g$ is homogeneous of degree 1. If $g=f$ then (2) yields $\dot{u}=f$ while (6) is not that simple except in the symmetric case $\gamma_{1}=\gamma_{2}$.

The difference between the two limiting equations can be interpreted in terms of particles in a variable environment. In the Lie-Trotter approach (2) the particle ensemble is subject to a changing environment (switching between two environments in the classical case) while in the situation of diffusive coupling (6) each particle switches between two phases according to Poisson processes with rates $\gamma_{1}, \gamma_{2}$. Another interpretation of (6) versus (2): In (6) the functions $f$ and $g$ act on the corresponding fraction of the total population while in (2) the weighted mean of $f$ and $g$ acts on the total population.

We connect the first order system for two variables (3) to a second order system for one variable (for smooth $f$ ). Differentiate the equations,

$$
\begin{aligned}
\ddot{v} & =f^{\prime}(v) \dot{v}-\gamma_{2} \dot{v}+\gamma_{1} \dot{w} \\
\ddot{w} & =g^{\prime}(w) \dot{w}-\gamma_{1} \dot{w}+\gamma_{2} \dot{v}
\end{aligned}
$$

and in the first equation replace $\dot{w}$ using the second equation of (3) and then replace $w$ from the first equation of (3). We get a second order system in $\mathbb{R}^{m}$ for the variable $v$,

$$
\tau \ddot{v}+\left(1-\tau f^{\prime}(v)\right) \dot{v}=\rho_{1} f(v)+\rho_{1} g\left(\left(\frac{\rho_{2}}{\rho_{1}}\right) v+\frac{\tau}{\rho_{1}}(\dot{v}-f(v))\right) .
$$

If $v$ is a solution of (7) then the solution of (3) can be recovered by putting $w=\left(\dot{v}-f(v)+\gamma_{2} v\right) / \gamma_{1}$. Hence (3) and (7) are equivalent.

In the limiting case of strong coupling, $\gamma_{i}=\gamma_{i} / \varepsilon, \varepsilon \rightarrow 0$, we get a first order system

$$
\dot{v}=\rho_{1} f(v)+\rho_{1} g\left(\frac{\rho_{2}}{\rho_{1}} v\right) .
$$

The function $u=v / \rho_{1}$ satisfies (6).

Example 1. For Verhulst equations $f(v)=a_{1} v\left(1-v / K_{1}\right), g(w)=a_{2} w(1-$ $\left.w / K_{2}\right)$ the limiting equation via diffusive coupling is

$$
\dot{u}=a u\left(1-\frac{u}{K}\right)
$$

where $a=\rho_{1} a_{1}+\rho_{2} a_{2}$ is the effective growth rate and

$$
K=\frac{\rho_{1} a_{1}+\rho_{2} a_{2}}{\frac{\rho_{1}^{2} a_{1}}{K_{1}}+\frac{\rho_{2}^{2} a_{2}}{K_{2}}}
$$


is the effective carrying capacity. The growth rate is an arithmetic mean of growth rates while the carrying capacity can be written as a harmonic mean of the carrying capacities times some factor independent of the $K_{i}$. The LieTrotter approach (2) yields the same value for the growth rate $a$ but the effective capacity is a simple harmonic mean

$$
K=\frac{\rho_{1} a_{1}+\rho_{2} a_{2}}{\frac{\rho_{1} a_{1}}{K_{1}}+\frac{\rho_{2} a_{2}}{K_{2}}} .
$$

Example 2. The ideas can be carried over to infinite-dimensional systems, e.g., reaction-diffusion equations. The system

$$
\begin{aligned}
v_{t} & =D \Delta v-\mu v-\gamma_{2} v+\gamma_{1} w \\
w_{t} & =f(w)-\gamma_{1} w+\gamma_{2} v
\end{aligned}
$$

describes a situation where the $v$ particles diffuse and are subject to mortality while the $w$ particles interact and do not move. In

$$
\begin{aligned}
v_{t} & =D \Delta v+f(v)-\gamma_{2} v+\gamma_{1} w \\
w_{t} & =-\gamma_{1} w+\gamma_{2} v
\end{aligned}
$$

a reaction-diffusion equation is coupled to a quiescent phase. For more details on coupled reaction-diffusion equations and quiescent transport equations see the references [11], [5], [6]. In [2] this approach is applied to a problem in protein dynamics. In [14] a coupled system has been used to understand the so-called river drift paradox in spread and persistence of species in stream ecosystems.

Example 3. The example of a delay equation $\dot{u}(t)=f(u(t-\theta))$ shows that one has to be careful with diffusive coupling. The system

$$
\begin{aligned}
\dot{v}(t) & =f(v(t-\theta))-\gamma_{2} v(t)+\gamma_{1} w(t) \\
\dot{w}(t) & =\gamma_{2} v(t)-\gamma_{1} w(t)
\end{aligned}
$$

may be of some interest but it is not the system one gets from coupling the dynamical system to the zero vector field. The reason is that the state space of the delay equation is not $\mathbb{R}$ but $C[-\tau, 0]$.

Example 4. Coupled dynamics is particularly relevant in epidemic modeling, when individuals switch between phases of different behavior, e.g., in corenon core situations or in public health education campaigns. In [4] coupling of moving and resting infected has been used to present the two classical approaches for epidemic spread, via diffusion and via contact distributions, within the same framework.

Again consider the case of $N \geq 2$ vector fields $f_{i}$ (notice that $v_{i}$ are vectors and not components of one vector) 


$$
\dot{v}_{i}=f_{i}\left(v_{i}\right)+\frac{1}{\tau} \sum_{k=1}^{N} \gamma_{i k} v_{k}
$$

The matrix $\Gamma=\left(\gamma_{i k}\right)$ has non-negative off-diagonal entries and column sums equal to 0 . Let $\Gamma$ be irreducible. Let $\rho=\left(\rho_{i}\right)$ with $\rho_{i}>0, \sum_{i=1}^{N} \rho_{i}=1$, be the unique stationary distribution. The "total particle density"

$$
u=\sum_{i=1}^{N} v_{i}
$$

satisfies the equation

$$
\dot{u}=\sum_{i=1}^{N} f_{i}\left(w_{i} u\right)
$$

whereby $w_{i}=v_{i} / u$. For $\tau \rightarrow 0$ the limiting equation becomes

$$
\dot{u}=\sum_{i=1}^{N} f_{i}\left(\rho_{i} u\right) .
$$

\subsection{Singular Perturbation Approach}

In the language of singular perturbation theory the system (5) is a slow system with $\tau$ as a small parameter [10]. Its solutions are called the outer solutions. Scaling the time variable as $\vartheta=t / \tau$ gives the fast system

$$
\begin{aligned}
& \dot{u}=\tau\left(f\left(\rho_{1} u+\tau z\right)+g\left(\rho_{2} u-\tau z\right)\right) \\
& \dot{z}=\rho_{2} f\left(\rho_{1} u+\tau z\right)-\rho_{1} g\left(\rho_{2} u-\tau z\right)-z
\end{aligned}
$$

which describes the dynamics in the initial layer, the so-called inner solution. It provides initial data for the outer solution. For the outer solution we solve the slow system (5) with initial conditions

$$
u(0)=u_{0}, \quad z(0)=\rho_{2} f\left(\rho_{1} u_{0}\right)-\rho_{1} g\left(\rho_{2} u_{0}\right) .
$$

We expand $u$ and $z$ as $u(t)=U_{0}(t)+\tau U_{1}(t)+\tau^{2} U_{2}(t)+\cdots$ and $z(t)=$ $Z_{0}(t)+\tau Z_{1}(t)+\tau^{2} Z_{2}(t)+\cdots$. To leading order we get from (5)

$$
\begin{aligned}
\dot{U}_{0} & =f\left(\rho_{1} U_{0}\right)+g\left(\rho_{2} U_{0}\right) \\
0 & =\rho_{2} f\left(\rho_{1} U_{0}\right)-\rho_{1} g\left(\rho_{2} U_{0}\right)-Z_{0} .
\end{aligned}
$$

Equation (12) describes the slow manifold

$$
\mathcal{M}_{0}=\left\{(u, z): z=\rho_{2} f\left(\rho_{1} u\right)-\rho_{1} g\left(\rho_{2} u\right)\right\}
$$

whereas the first equation (11) describes the dynamics on that manifold. Note that this dynamics is the same as in (6) whereby the formal limit (8) is 
justified. The inner solution approximates the slow manifold $\mathcal{M}_{0}$ as $\vartheta \rightarrow \infty$ and then the matching initial conditions in $\mathcal{M}_{0}$ as given in (10).

To apply Fenichel's geometric singular perturbation theory ([10]) we show that $\mathcal{M}_{0}$ is normally hyperbolic.

Lemma 1. The manifold $\mathcal{M}_{0}$ is normally hyperbolic with respect to the flow of the fast system at $\tau=0$.

Proof. Linearize the fast system at $(\bar{u}, \bar{z}) \in \mathcal{M}_{0}$,

$$
\frac{d}{d \vartheta}\left(\begin{array}{l}
u \\
z
\end{array}\right)=A_{\tau}\left(\begin{array}{l}
u \\
z
\end{array}\right) \quad \text { with } \quad A_{0}=\left(\begin{array}{cc}
0 & 0 \\
a & -I_{m}
\end{array}\right)
$$

whereby $a=\rho_{1} \rho_{2}\left(f^{\prime}\left(\rho_{1} \bar{u}\right)-g^{\prime}\left(\rho_{2} \bar{u}\right)\right)$, and $f^{\prime}$ and $g^{\prime}$ denote the Jacobians of $f, g$ and $I_{m}$ is the $m \times m$ identity. Hence the eigenvalues are 0 and -1 with eigenvectors $(0,1)^{T}$ and $(1, a)^{T}$.

Fenichel's first theorem (Theorem 1 and 2 in [10]) gives

Theorem 2. For $\tau>0$ small enough there exists a locally invariant manifold $\mathcal{M}_{\tau}$ (the critical manifold) with the following properties:

i) $\mathcal{M}_{\tau}$ is $\tau$-close to $\mathcal{M}_{0}$.

ii) $\mathcal{M}_{\tau}$ is locally invariant for the fast system.

iii) $\mathcal{M}_{\tau}=\left\{(u, z): z=h^{\tau}(u)\right\}$ is a graph with $h^{0}(u)=\rho_{2} f\left(\rho_{1} u\right)-\rho_{1} g\left(\rho_{2} u\right)$.

From the linearization in Lemma 1 one sees that $\mathcal{M}_{0}$ has a stable manifold of dimension $m$. From Fenichel's second theorem (Theorem 3 in [10]) it follows that $\mathcal{M}_{\tau}$ has an $m$-dimensional stable manifold and solutions approach $\mathcal{M}_{\tau}$ exponentially fast. In the present case the exponent is -1 .

\subsection{Global Behavior}

We cannot expect that the dynamics of the coupled system (3) is largely determined by the dynamics of the two constituents (15), not even in the linear case $f(v)=A v, g(w)=B w$ there are useful results for general matrices $A, B$. The only immediate observation is concerned with gradient fields.

Proposition 1. Assume the vector fields $f$ and $g$ are gradient fields. Then the system (3) is equivalent to a system with a gradient field.

Proof. By substituting $v=\sqrt{\gamma_{1}} \tilde{v}, w=\sqrt{\gamma_{2}} \tilde{w}$ and then dropping the tildes the system (3) becomes

$$
\begin{gathered}
\dot{v}=\frac{1}{\sqrt{\gamma_{1}}} f\left(\sqrt{\gamma_{1}} v\right)-\gamma_{2} v+\sqrt{\gamma_{1} \gamma_{2}} w \\
\dot{w}=\frac{1}{\sqrt{\gamma_{2}}} g\left(\sqrt{\gamma_{2}} w\right)-\gamma_{1} w+\sqrt{\gamma_{1} \gamma_{2}} v .
\end{gathered}
$$


Let $f=F^{\prime}, g=G^{\prime}$. Then (13) has the potential

$$
\frac{1}{\gamma_{1}} F\left(\sqrt{\gamma_{1}} v\right)+\frac{1}{\gamma_{2}} G\left(\sqrt{\gamma_{2}} w\right)-\frac{1}{2}\left\|\sqrt{\gamma_{2}} v-\sqrt{\gamma_{1}} w\right\|^{2}
$$

Even this result is not generally applicable: If $F, G$ are bounded below then the potential (14) need not be bounded below. To compensate for the negative term for large $w$ the functionals $F, G$ must grow at least quadratically. However, the result is strong enough for space dimension one.

Corollary 1. If the space dimension is $m=1$ then every bounded trajectory of (3) converges to an equilibrium.

This result could also have been concluded from the theory of cooperative systems [7] since for $m=1$ the system (3) is cooperative. Our next observations are concerned with global attractors.

Suppose it is known that the limiting system (6) has a (local or global) attractor. We will show that, for $\tau$ small enough, the system (9) has a local attractor as well. Let $\mathcal{A}_{0}$ denote the attractor of (6) in question. Then define

$$
\tilde{\mathcal{A}}_{0}=\left\{(u, z): u \in A_{0}, z=h^{0}(u)\right\}
$$

where $h^{0}(u)$ has been defined in Theorem 2. From the general theory of attractors (see, e.g. Temam [18] or Robinson [16]) we obtain the following result.

Theorem 3. Assume the system (6) in $\mathbb{R}^{m}$ has a compact local or global attractor $\mathcal{A}_{0}$. For $\tau>0$ small enough the system (9) in $\mathbb{R}^{2 m}$ has a compact attractor $\mathcal{A}_{\tau} \subset \mathbb{R}^{2 m}$ near $\tilde{\mathcal{A}}_{0}$ in the sense that $\mathcal{A}_{\tau}$ is upper semi-continuous at $\tilde{\mathcal{A}}_{0}$ for $\tau=0$, i.e., $\lim _{\tau \rightarrow 0} \operatorname{dist}\left(\mathcal{A}_{\tau}, \tilde{\mathcal{A}}_{0}\right)=0$ where

$$
\operatorname{dist}(X, Y)=\sup _{x \in X} \inf _{y \in Y}\|x-y\| \text {. }
$$

Moreover, if in addition

$$
\mathcal{A}_{0}=\cup_{\xi \in \mathcal{E}} \overline{W^{u}(\xi)}
$$

where $\mathcal{E}$ consists of a finite number of equilibria, then the attractors are lower semi-continuous as well, i.e. $\lim _{\tau \rightarrow 0} \operatorname{dist}\left(\tilde{\mathcal{A}}_{0}, \mathcal{A}_{\tau}\right)=0$.

Note that in Theorem 3 the attractor $\mathcal{A}_{\tau}$ need not be a global attractor, see the following example.

Example 5. Let $m=1, \rho_{1}=\rho_{2}, f(x)=x(x-1), g(x)=-x(x+1)$. Then the limiting system (6) reads $\dot{u}=-u$ which has the compact global attractor $\mathcal{A}_{0}=\{0\}$. The coupled system (5) reads

$$
\dot{u}=(-1+\tau z), \quad \tau \dot{z}=\frac{u^{2}}{4}+(\tau z)^{2}-2 z .
$$

From the second equation it is evident that solutions with large $z(0)>0$ blow up in finite time. But $\mathcal{A}_{\tau}\{(0,0)\}=\tilde{\mathcal{A}}_{0}$ is an asymptotically stable node and hence it is still a local attractor. 


\section{Quiescent Phases}

Probably the most interesting special case of equation (3) arises if the vector field $g$ vanishes. Then we can interpret $w$ as a resting or quiescent phase. Let the dynamics in $\mathbb{R}^{m}$ be given by

$$
\dot{u}=f(u) \text {. }
$$

Then the corresponding system with quiescent phase is

$$
\begin{aligned}
\dot{v} & =f(v)-\gamma_{2} v+\gamma_{1} w \\
\dot{w} & =-\gamma_{1} w+\gamma_{2} v
\end{aligned}
$$

and the second order system (7) reads $\tau \ddot{v}+\left(1-\tau f^{\prime}(v)\right) \dot{v}=\rho_{1} f(v)$. For $\tau \rightarrow 0$ we recover the dynamics (15) with a different time scale. Also the limiting system (6) recovers the original system (15) on a different time scale $\dot{u}=f\left(\rho_{1} u\right)$.

Example 6. If the Verhulst equation $\dot{u}=a u(1-u / K)$ is coupled to a quiescent phase, then the limiting equation reads

$$
\dot{u}=a \rho_{1} u\left(1-\rho_{1} \frac{u}{K}\right)
$$

The exponent is decreased to $a \rho_{1}$ and the carrying capacity is increased to $K / \rho_{1}$ saying that the population grows slower and the habitat can support a larger population.

The equation with Allee effect $\dot{u}=u(1-u)(u-\alpha)$, with $0<\alpha<1$, if coupled to a quiescent phase, yields the limiting equation

$$
\dot{u}=\rho_{1} u\left(1-\rho_{1} u\right)\left(\rho_{1} u-\alpha\right) .
$$

The carrying capacity is increased and also the threshold.

\subsection{Stabilization by Quiescent Phases}

Introducing a quiescent phase does not essentially change the equilibria.

Lemma 2. The stationary points of the system (16) with quiescent phase are essentially the same as those of the simple dynamics (15), i.e., they have the form $\left(\bar{v},\left(\gamma_{2} / \gamma_{1}\right) \bar{v}\right)$ where $f(\bar{v})=0$.

In the view of interacting particles a quiescent phase should act as a delay. Introducing a delay into a dynamical system with negative feedback in general causes oscillatory instability if the delay is large. But introducing a quiescent phase does not lead to oscillatory instability, quite on the contrary, it is stabilizing. This fact can be seen from the following theorem which has been proved in [3]. In (16), with $m \geq 1$, assume $f(\bar{v})=0$. Then $(\bar{v}, \bar{w})$ with $\bar{w}=\gamma_{2} \bar{v} / \gamma_{1}$ is a 
stationary state. The eigenvalues $\mu$ of $f^{\prime}(\bar{v})$ and the corresponding eigenvalues $\lambda$ of the Jacobian at $(\bar{v}, \bar{w})$ are connected by the equation

$$
\varphi(\lambda) \equiv \lambda^{2}+\lambda\left(\gamma_{1}+\gamma_{2}-\mu\right)-\mu \gamma_{1}=0 .
$$

We describe the relationships between these eigenvalues in detail.

Theorem 4. Let $\mu$ be an eigenvalue of the linearization of (15) at a steady state $\bar{u}$. Then the linearization of (16) at $\left(\bar{u}, \gamma_{2} \bar{u} / \gamma_{1}\right)$ has two corresponding eigenvalues $\lambda_{1}, \lambda_{2}$ with $\Re \lambda_{2} \leq \Re \lambda_{1}$. The eigenvalues $\mu$ and $\lambda_{1}, \lambda_{2}$ are related as follows:

(a) Let $\mu=\alpha \in \mathbb{R}$. Then $\lambda_{1}, \lambda_{2}$ are real.

(a.i) If $\alpha<0$ then $\lambda_{2}<\alpha<\lambda_{1}<0$.

(a.ii) If $\alpha=0$ then $\lambda_{2}=-\left(\gamma_{1}+\gamma_{2}\right)<0=\lambda_{1}$.

(a.iii) If $\alpha>0$ then $\lambda_{2}<0<\lambda_{1}<\alpha$.

(b) Let $\mu=\alpha \pm i \beta, \beta>0$. Then $\Re \lambda_{2}<0$.

(b.i) If $\alpha \leq 0$ then $\Re \lambda_{1}<0$.

(b.ii) If $\alpha>0$ then $\Re \lambda_{1}<\alpha$.

(b.iii) If $\alpha \leq 0$ and

$$
\beta^{2}+\left(\gamma_{1}+\gamma_{2}+\alpha\right)^{2}+4 \alpha \gamma_{2}>0 \text { and } \beta^{2}\left(\gamma_{1}+\alpha\right)+\alpha\left(\gamma_{1}+\gamma_{2}+\alpha\right)^{2}>0,
$$

then $\Re \lambda_{1}<\alpha$.

(b.iv) If $\alpha>0$ and

$$
\beta^{2}>4 \alpha \gamma_{1}-\left(\gamma_{1}+\gamma_{2}-\alpha\right)^{2} \text { and } \beta\left(\gamma_{2}-\alpha\right)>\alpha\left(\gamma_{1}+\gamma_{2}-\alpha\right)^{2} \text {, }
$$

then $\Re \lambda_{1}<0$.

With respect to the leading eigenvalue $\lambda_{1}$ the theorem says that a zero eigenvalue is maintained while non-zero real eigenvalues maintain their sign and move closer towards zero. For conjugate complex eigenvalues in general the real part decreases, in particular if the imaginary part is large (in absolute value). Purely imaginary eigenvalues are always carried into eigenvalues with negative real parts. The property (b.i) was also proven in Neubert et al. [13]. Properties (b.iii) and (b.iv) say that if $\gamma_{i}>>|\alpha|$ and $\beta^{2}$ is large then oscillations are damped.

A proof of Theorem 4 is given in [3].

Example 7 (Paradox of enrichment). The MacArthur-Rosenzweig model for a prey-predator population exhibits a Hopf bifurcation when the capacity of the prey exceeds a certain threshold. We extend the model by a quiescent phase for the prey only. The extended system for active prey $x$, predators $y$ and quiescent prey $z$ reads

$$
\begin{aligned}
& \dot{x}=a x\left(1-\frac{x}{K}\right)-\frac{b x y}{A+x}-\gamma_{2} x+\gamma_{1} z \\
& \dot{y}=c y\left(\frac{x}{A+x}-\frac{B}{B+A}\right) \\
& \dot{z}=\gamma_{2} x-\gamma_{1} z .
\end{aligned}
$$


There are stationary states $(0,0,0),(K, 0,0)$ and the coexistence state $(\bar{x}, \bar{y}, \bar{z})$ with $\bar{x}=B, \bar{y}=(a / b)(1-B / K)(A+B), \bar{z}=\gamma_{2} B / \gamma_{1}$. Notice that $\bar{x}, \bar{y}$ do not depend on $\gamma_{1}, \gamma_{2}$. The coexistence state is feasible (positive) if $B<K$. In the absence of a quiescent phase the coexistence state is stable if $B>(K-A) / 2$ and unstable if $B<(K-A) / 2$.

The characteristic polynomial of the Jacobian at the coexistence state is

$$
\lambda^{3}+\left(\gamma_{1}+\gamma_{2}-T\right) \lambda^{2}+\left(S-T \gamma_{1}\right) \lambda+S \gamma_{1}=0
$$

where

$$
T=\frac{a B}{K(A+B)}(K-A-2 B), \quad S=\frac{a c A B}{K(A+B)^{2}}(K-B) .
$$

Because of $S>0$ the Routh-Hurwitz criterion for stability reduces to the single inequality

$$
\left(\gamma_{1}+\gamma_{2}-T\right)\left(S-\gamma_{1} T\right)>S \gamma_{1} .
$$

For the system with a quiescent phase we have stability for $T<0$. In that case also (17) is satisfied for any choice of $\gamma_{1}, \gamma_{2}>0$. On the other hand, if $T>0$ then the system can be stabilized by first choosing $\gamma_{1}<S / T$ and then $\gamma_{2}$ so large that (17) is satisfied. Hence the system can be stabilized by making the exit rate from the quiescent compartment small and the entrance rate to that compartment large. We find that the system becomes stabilized against oscillations when the prey has a refuge in the form of a quiescent phase.

\subsection{Periodic Orbits and Quiescent Phases}

We know that near a stationary point a quiescent phase tends to suppress oscillations. Now we ask what effect a quiescent phase has on existing "large" periodic orbits expecting that in some sense the "amplitude" is decreased or that the periodic orbit disappears. We choose the model problem in the plane

$$
\dot{r}=r g(r), \quad \dot{\varphi}=1
$$

where $g \in C^{1}(\mathbb{R})$ is strictly decreasing from $g(0)>0$ to negative values, and $g(\bar{r})=0, g^{\prime}(\bar{r})<0$. In cartesian coordinates the system reads, with $r^{2}=u_{1}^{2}+u_{2}^{2}$,

$$
\begin{aligned}
& \dot{u}_{1}=g(r) u_{1}-u_{2} \\
& \dot{u}_{2}=g(r) u_{2}+u_{1} .
\end{aligned}
$$

We define a system with an active phase $v$ and a quiescent phase $w$ as before (a system in $\mathbb{R}^{4}$ ). We return to polar coordinates $v=(r, \varphi), w=(\rho, \psi)$ and get a four-dimensional system 


$$
\begin{aligned}
\dot{r} & =r g(r)-\gamma_{2} r+\gamma_{1} \rho \cos (\varphi-\psi) \\
\dot{\varphi} & =1-\gamma_{1}(\rho / r) \sin (\varphi-\psi) \\
\dot{\rho} & =-\gamma_{1} \rho+\gamma_{2} r \cos (\varphi-\psi) \\
\dot{\psi} & =\gamma_{2}(r / \rho) \sin (\varphi-\psi) .
\end{aligned}
$$

Finally we introduce the displacement angle $\theta=\varphi-\psi$ and get a threedimensional system

$$
\begin{aligned}
& \dot{r}=r g(r)-\gamma_{2} r+\gamma_{1} \rho \cos \theta \\
& \dot{\theta}=1-\left(\gamma_{1}(\rho / r)+\gamma_{2}(r / \rho)\right) \sin \theta \\
& \dot{\rho}=-\gamma_{1} \rho+\gamma_{2} r \cos \theta .
\end{aligned}
$$

For this system we prove the following result.

Theorem 5. Consider the model problem (19). Define $\bar{\beta}=\bar{\beta}\left(\gamma_{1}, \gamma_{2}\right)$ as the unique positive solution of the cubic equation

$$
\beta\left(\beta-\gamma_{2}-\gamma_{1}\right)^{2}+\beta-\gamma_{2}=0,
$$

and let $\bar{\kappa}$ be the unique positive solution of the equation

$$
\frac{\gamma_{1}^{2}}{\gamma_{2}^{2}} \kappa+\frac{\kappa}{\left(\gamma_{1} \kappa+\gamma_{2}\right)^{2}}=1
$$

If $g(0)>\bar{\beta}$ then let $R$ be defined by $g(R)=\bar{\beta}$. In this case the system has a periodic orbit with constant radius $r=R<\bar{r}$, whereby $\rho=R \bar{\kappa}$. If $g(0) \leq \bar{\beta}$ then there is no such orbit.

Proof. We look for a solution of (19) along which $r, \rho$ and $\theta$ are constant. Then the time derivatives are zero and we have three equations for $r, \rho, \theta$. From the last two equations we find

$$
\cos \theta=\frac{\gamma_{1} \rho}{\gamma_{2} r}, \quad \sin \theta=\left(\gamma_{1} \frac{\rho}{r}+\gamma_{2} \frac{r}{\rho}\right)^{-1}
$$

and hence we get two equations for $r$ and $\kappa=(\rho / r)^{2}$,

$$
g(r)-\gamma_{2}+\frac{\gamma_{1}^{2}}{\gamma_{2}} \kappa=0,
$$

and (21). If $\kappa$ runs from 0 to $\infty$ then the left hand side of (21) is strictly increasing from 0 to $\infty$. Hence there is a unique solution $\bar{\kappa}>0$ which depends only on $\gamma_{1}$ and $\gamma_{2}$. Furthermore the left hand side of (21) is greater than 1 at $\kappa=\gamma_{2}^{2} / \gamma_{1}^{2}$, hence we have

$$
\bar{\kappa}\left(\gamma_{1}, \gamma_{2}\right)<\frac{\gamma_{2}^{2}}{\gamma_{1}^{2}}
$$


Now $\bar{\kappa}$ has been determined and equation (22) becomes $g(r)=\bar{\beta}$ whereby

$$
\bar{\beta}=\gamma_{2}-\frac{\gamma_{1}^{2}}{\gamma_{2}} \bar{\kappa}\left(\gamma_{1}, \gamma_{2}\right) .
$$

We have $\bar{\beta}>0$ in view of (23).

Now we express $\bar{\kappa}$ in terms of $\bar{\beta}$ which gives $\bar{\kappa}=\left(\gamma_{2}-\bar{\beta}\right) \gamma_{1} / \gamma_{1}^{2}$, we insert this expression into (21), and get an equation for $\bar{\beta}$ in terms of $\gamma_{1}, \gamma_{2}$ which is (20). This equation has a unique positive solution.

Now there are two cases.

Case 1: $g(0)>\bar{\beta}$. Then there is a unique value $R$ with $g(R)=\bar{\beta}$. Determine $\bar{\theta}$ from

$$
\cos \bar{\theta}=\left(\gamma_{1} / \gamma_{2}\right) \sqrt{\bar{\kappa}}, \quad\left(\gamma_{1} \sqrt{\bar{\kappa}}+\gamma_{2}(1 / \sqrt{\bar{\kappa}})\right) \sin \bar{\theta}=1 .
$$

Finally go back to (18) and find a solution $r=R, \rho=\sqrt{\bar{\kappa}} R, \dot{\varphi}=\dot{\psi}=$ $1-\gamma_{1} \sqrt{\bar{\kappa}} \sin \bar{\theta}$. The equation (21) for $\kappa=\bar{\kappa}$ ensures that indeed $\dot{\varphi}=\dot{\psi}$. The period of the orbit is $T=2 \pi\left(\gamma_{1} \bar{\kappa} / \gamma_{2}+1\right)$.

Case 2: $g(0) \leq \bar{\beta}$. Then the equation $g(r)=\bar{\beta}$ has no solution.

\subsection{Global Behavior of Systems with Quiescent Phases}

We further explore the connection between (15) and (16). If (15) has a compact global attractor then one can ask whether (16) has a compact global attractor. Such a general result is perhaps not true. We show a weaker result using the Lyapunov function $v^{T} v$. Even for space dimension 1 the problem of global existence is not trivial.

Lemma 3. Let $m=1$. Assume that all solutions of (15) exist for all times $t \geq 0$. Then all solutions of (16) exist for all positive times.

Proof. Assume that $(v, w) \rightarrow \infty$ in finite time. Then $v \rightarrow \infty$ in finite time. For if $v$ would stay bounded then $w$ would be bounded in finite time (variation of constants applied to the second equation of (16)). Since the system is cooperative, the tangent vector $(\dot{v}, \dot{w})$ can switch the orthant at most once and eventually stays in one of the four orthants [7]. We distinguish several cases according to the orthant.

Case 1: $\dot{v} \geq 0, \dot{w} \geq 0$. Let $v \rightarrow \infty$ in finite time. Then $\gamma_{1} v-\gamma_{2} w \geq 0$ and $\dot{v} \leq f(v)$. Hence $v(t) \leq u(t)$ where $u$ is the solution of (15) with the same initial value. Hence $u \rightarrow \infty$ in finite time which is a contradiction.

Case 2: $\dot{v} \geq 0, \dot{w}<0$. Let $v$ go to infinity in finite time. From

$$
\dot{w}(t)=\left(\gamma_{2} v(0)-\gamma_{1} w(0)+\gamma_{2} \int_{0}^{t} e^{\gamma_{1} s} \dot{v}(s) d s\right) e^{-\gamma_{1} t}
$$

it follows that $\dot{w}$ becomes eventually positive. Hence this case is impossible. The preceding formula follows easily from $\ddot{w}=\gamma_{2} \dot{v}-\gamma_{1} \dot{w}$, but it holds also if $w$ is only $C^{1}$.

The other two orthants are treated similarly, $\dot{v} \leq 0, \dot{w} \leq 0$ as in case $1, \dot{v} \leq 0$, $\dot{w} \geq 0$ as in case 2 . 
We do not know whether a similar statement holds in several dimensions. However, we show the following:

Theorem 6. Let $v^{T} f(v)<0$ hold for large $\|v\|$. Then for any choice of $\gamma_{1}, \gamma_{2}>0$ the system (16) has a compact global attractor.

Proof. We show that for some large $R$ the ball $\gamma_{2} v^{2}+\gamma_{1} w^{2} \leq R^{2}$ is positively invariant. We write $v^{2}=v^{T} v$. Along trajectories of (16) we have

$$
\frac{1}{2} \frac{d}{d t}\left(\gamma_{2} v^{2}+\gamma_{1} w^{2}\right)=\gamma_{2} v^{T} f(v)-\left(\gamma_{2} v-\gamma_{1} w\right)^{T}\left(\gamma_{2} v-\gamma_{1} w\right)
$$

Choose $r>0$ such that $v^{T} f(v)<0$ for $\|v\| \geq r$. Choose $m>0$ such that $v^{T} f(v) \leq m$ for $\|v\| \leq r$. Finally choose $R>\sqrt{\left(\sqrt{\gamma_{2} m}+\gamma_{2} r\right)^{2} / \gamma_{1}+\gamma_{2} r^{2}}$. We want to show $\frac{1}{2} d / d t\left(\gamma_{2} v^{2}+\gamma_{1} w^{2}\right)<0$ for $\gamma_{2} v^{2}+\gamma_{1} w^{2} \geq R^{2}$. Let $(v, w)$ such that $\gamma_{2} v^{2}+\gamma_{1} w^{2} \geq R^{2}$.

Case 1: $\|v\| \geq r$. Then $v^{T} f(v)<0$ by assumption and hence the desired inequality is evident.

Case 2: $\|v\|<r$. From $\gamma_{2} v^{2}+\gamma_{1} w^{2} \geq R^{2}$ it follows that

$$
w^{2} \geq \frac{1}{\gamma_{1}}\left(R^{2}-\gamma_{2} v^{2}\right) \geq \frac{1}{\gamma_{1}}\left(R^{2}-\gamma_{2} r^{2}\right)
$$

and the right hand side is positive by assumption on $R$. Hence

$$
\begin{gathered}
\|w\| \geq \frac{1}{\sqrt{\gamma_{1}}} \sqrt{R^{2}-\gamma_{2} r^{2}} \\
\left\|\gamma_{1} w-\gamma_{2} v\right\| \geq \gamma_{1}\|w\|-\gamma_{2}\|v\| \geq \sqrt{\gamma_{1}} \sqrt{R^{2}-\gamma_{2} r^{2}}-\gamma_{2} r .
\end{gathered}
$$

Finally

$$
\frac{1}{2} \frac{d}{d t}\left(\gamma_{2} v^{2}+\gamma_{1} w^{2}\right) \leq \gamma_{2} m-\left[\sqrt{\gamma_{1}} \sqrt{R^{2}-\gamma_{2} r^{2}}-\gamma_{2} r\right]^{2}
$$

which is negative by the assumption on $R$.

Corollary 2. Let the space dimension be $m=1$. If the original system (15) has a bounded global attractor then for any choice of $\gamma_{1}, \gamma_{2}$ the system with a quiescent phase (16) has a bounded global attractor. This global attractor consists of the stationary states and their unstable manifolds.

Proof. In space dimension 1 the condition $v f(v)<0$ for large $|v|$ is necessary and sufficient for the existence of a global attractor. The remainder of the proof follows from Theorem 6 . 


\section{Proof of Theorem 1}

The difference $u_{\delta}-u$ satisfies

$$
\begin{aligned}
\dot{u}_{\delta}-\dot{u} & =\chi(t / \delta) f\left(u_{\delta}\right)+(1-\chi(t / \delta)) g\left(u_{\delta}\right)-\rho_{1} f(u)-\left(1-\rho_{1}\right) g(u) \\
& =\chi(t / \delta) f\left(u_{\delta}\right)+(1-\chi(t / \delta)) g\left(u_{\delta}\right)-\chi(t / \delta) f(u)-(1-\chi(t / \delta)) g(u) \\
& +\chi(t / \delta) f(u)+(1-\chi(t / \delta)) g(u)-\rho_{1} f(u)-\left(1-\rho_{1}\right) g(u) \\
& =\chi(t / \delta)\left(f\left(u_{\delta}\right)-f(u)\right)+(1-\chi(t / \delta))\left(g\left(u_{\delta}\right)-g(u)\right) \\
& +\left(\chi(t / \delta)-\rho_{1}\right)(f(u)-g(u)) .
\end{aligned}
$$

We integrate

$$
\begin{aligned}
u_{\delta}(t)-u(t) & =\int_{0}^{t}\left[\chi(s / \delta)\left(f\left(u_{\delta}\right)-f(u)\right)+(1-\chi(t / \delta))\left(g\left(u_{\delta}\right)-g(u)\right)\right] d s \\
& +\int_{0}^{t}\left(\chi(s / \delta)-\rho_{1}\right)(f(u)-g(u)) d s+u_{\delta}(0)-u(0)
\end{aligned}
$$

and get a first estimate

$$
\begin{aligned}
\left\|u_{\delta}(t)-u(t)\right\| & \leq\left\|u_{\delta}(0)-u(0)\right\|+2 M \int_{0}^{t}\left\|u_{\delta}-u\right\| d s \\
& +\left\|\int_{0}^{t}\left(\chi(t / \delta)-\rho_{1}\right) \phi(s) d s\right\|
\end{aligned}
$$

where $\phi$ is the fixed continuous vector function $\phi(s)=f(u(s))-g(u(s))$. The other factor in the integral is a scalar function which is rapidly oscillating around zero. Hence the integral should be small. It is sufficient to show this for the case where $\phi$ is a scalar function.

Let $\epsilon>0$ be given. Choose a step function $\psi(s)$ with finitely many steps such that

$$
|\phi(s)-\psi(s)|<\epsilon e^{-2 M T} / 2
$$

for $0 \leq s \leq T$. Let $N=N(\epsilon)$ be the number of steps, let the $N$ step intervals be denoted by $I_{k}, k=1, \ldots, N$, and the values of the step function by $\psi_{k}$.

Then choose $t \in[0, T]$ fixed. Let $N^{\prime} \leq N$ be the largest number such that $I_{k} \cap[0, T) \neq \emptyset$ for $k=1, \ldots, N^{\prime}$, and let $I_{k}^{\prime}=I_{k} \cap[0, t]$. Of course $I_{k}^{\prime}=I_{k}$ for $k<N^{\prime}$. Then we have

$$
\int_{0}^{t}\left(\chi(s / \delta)-\rho_{1}\right) \psi(s) d s=\sum_{k=1}^{N^{\prime}} \int_{I_{k}^{\prime}}\left(\chi(s / \delta)-\rho_{1}\right) d s \psi_{k} .
$$

Consider the $k$-th step. The length of the $k$-th interval can be expressed in multiples of $\delta$ as $\left|I_{k}^{\prime}\right|=n_{k} \delta+r_{k}$ with $0 \leq r_{k}<\delta$. Since the integral of $\chi(s / \delta)-\rho_{1}$ over an interval of length $\delta$ vanishes, from the $k$-th term remains only an integral over an interval of length $r_{k}$ which can be estimated by $r_{k}\left|\psi_{k}\right| \leq \delta 2 M$. Hence 


$$
\left|\int_{0}^{t}\left(\chi(s / \delta)-\rho_{1}\right) \psi(s) d s\right| \leq 2 M N^{\prime} \delta \leq 2 M N \delta .
$$

Now choose $\delta_{0}$ such that

$$
4 M N e^{2 M T} \delta_{0}<\epsilon
$$

Then

$$
\left|\int_{0}^{t}\left(\chi\left(\frac{s}{s}\right)-\rho_{1}\right) \psi(s) d s\right| \leq \epsilon e^{-2 M T} / 2
$$

for all $\delta<\delta_{0}$ and hence (returning to the vector case)

$$
\left\|\int_{0}^{t}\left(\chi(s / \delta)-\rho_{1}\right) \phi(s) d s\right\| \leq \epsilon e^{-2 M T}
$$

for all $\delta<\delta_{0}$. From (24) we find

$$
\left\|u_{\epsilon}(t)-u(t)\right\| \leq\left\|u_{\epsilon}(0)-u(0)\right\|+2 M \int_{0}^{t}\left\|u_{\epsilon}-u\right\| d s+\epsilon e^{-2 M T} .
$$

A Gronwall argument gives

$$
\int_{0}^{t}\left\|u_{\epsilon}(s)-u(s)\right\| d s \leq \frac{\left\|u_{\epsilon}(0)-u(0)\right\|+\epsilon e^{-2 M T}}{2 M}\left(e^{2 M T}-1\right) .
$$

Introduce this expression into (25) and get

$$
\left\|u_{\epsilon}(t)-u(t)\right\| \leq\left\|u_{\epsilon}(0)-u(0)\right\| e^{2 M T}+\epsilon .
$$

Hence, for $u_{\epsilon}(0)=u(0)$ we get for $0 \leq t \leq T$

$$
\left\|u_{\epsilon}(t)-u(t)\right\| \leq \epsilon
$$

which shows uniform convergence for $\epsilon \rightarrow 0$.

\section{References}

1. R. Bathia, Matrix Analysis, Springer 1996.

2. G. Carrero, D. McDonald, E. Crawford, G. de Vries, and M. Hendzel, Using FRAP and mathematical modeling to determine the in vivo kinetics of nuclear proteins. Methods, 29:14-28, 2003.

3. K.P. Hadeler, Quiescent phases and stability. submitted.

4. K.P. Hadeler, The role of migration and contact distribution in epidemic spread. In C. Castillo-Chavez and H.T. Banks, editors, Frontiers Appl. Math., 28, Bioterrorism, pages 188-210. SIAM, 2003.

5. K.P. Hadeler and M.A. Lewis, Spatial dynamics of the diffusive logistic equation with a sedentary compartment. Canadian Appl. Math. Quart., 10:473-499, 2002.

6. T. Hillen, Transport equations with resting phases. Europ. J. Appl. Math., 14:613-636, 2003. 
7. M.W. Hirsch, H.L. Smith, Monotone dynamical systems. pp. 239-357 In: Handbook of Differential Equations. Ordinary Differential Equations, Vol. 2 (eds. A Cañada, P. Drábek A. Fonda) Elsevier 2005.

8. R.A. Horn, C. Johnson, Topics in Matrix Analysis. Cambridge U. Press 1994.

9. W. Jäger, S. Krömker, and B. Tang, Quiescence and transient growth dynamics in chemostat models. Math. Biosci. 119:225-239, 1994.

10. C.K.R.T. Jones, Geometric singular perturbation theory. In J. Russell, editor, Dynamical Systems, CIME Lectures Montecatini Terme, Italy, 1994. Lect. Notes Math. 1609, 44-118 1995.

11. M.A. Lewis and G. Schmitz, Biological invasion of an organism with separate mobile and stationary states: Modeling and analysis. Forma, 11:1-25, 1996.

12. T. Malik, H.L. Smith, A resource-based model of microbial quiescence. J. Math. Biol., 53, 231-252, 2006

13. M.G. Neubert, P. Klepac, and P. van den Driessche, Stabilizing dispersal delays in predator-prey metapopulation models. Theor. Popul. Biol., 61:339-347, 2002.

14. E. Pachepsky, F. Lutscher, R.M. Nisbet, M. Lewis, Persistence, spread and the drift paradox. Theor. Pop. Biol. 67:61-73, 2005.

15. A. Pazy, Semigroups of Linear Operators and Applications to Partial Differential Equations. Springer, New York, 1983.

16. J.C. Robinson, Infinite-Dimensional Dynamical Systems. Cambridge Texts in Applied Mathematics. Cambridge University Press, Cambridge, 2001.

17. B. Tang, Mathematical models of microbial competition in laboratory cultures incorporating environmental heterogeneities: coexistence. Surveys Math. Indust. 3, 49-70, 1993.

18. R. Temam, Infinite-Dimensional Dynamical systems in Mechanics and Physics. Springer, 1988.

19. G. Webb, Structured population dynamics. Mathematical modelling of population dynamics, 123-163, Banach Center Publ., 63, Polish Acad. Sci., Warsaw, 2004 . 